\title{
Pacific
}

Journal of

Mathematics

\section{A CONVERSE TO A THEOREM OF KOMLÓS FOR CONVEX SUBSETS OF $L_{1}$}

\author{
CHRISTOPHER JOHN LENNARD
}




\title{
A CONVERSE TO A THEOREM OF KOMLÓS FOR CONVEX SUBSETS OF $L_{1}$
}

\section{Chris LenNARD}

\begin{abstract}
A theorem of Komlós is a subsequence version of the strong law of large numbers. It states that if $\left(f_{n}\right)_{n}$ is a sequence of norm-bounded random variables in $L_{1}(\mu)$, where $\mu$ is a probability measure, then there exists a subsequence $\left(g_{k}\right)_{k}$ of $\left(f_{n}\right)_{n}$ and $f \in L_{1}(\mu)$ such that for all further subsequences $\left(h_{m}\right)_{m}$, the sequence of successive arithmetic means of $\left(h_{m}\right)_{m}$ converges to $f$ almost everywhere.

In this paper we show that, conversely, if $C$ is a convex subset of $L_{1}(\mu)$ satisfying the conclusion of Komlós' theorem, then $C$ must be $L_{1}$-norm bounded.
\end{abstract}

Introduction. A version of the strong law of large numbers in probability theory states that if $\left(f_{n}\right)_{n=1}^{\infty}$ is a sequence of independent, scalarvalued integrable functions (random variables), on a probability measure space $(\Omega, \Sigma, \mu)$, each having the same distribution with mean $m$, then

$$
\frac{1}{n} \sum_{j=1}^{n} f_{j} \rightarrow m \text { almost everywhere. }
$$

In (1967) Komlós [Ko] showed that arbitrary sequences of integrable random variables whose absolute values have uniformly bounded expectations always have subsequences that satisfy a version of the strong law. Indeed, for all sequences $\left(f_{n}\right)_{n=1}^{\infty}$ in $L_{1}(\mu)$ with

$$
\sup _{n} \int_{\Omega}\left|f_{n}\right| d \mu<\infty
$$

there exists a subsequence $\left(g_{k}\right)_{k=1}^{\infty}$ of $\left(f_{n}\right)_{n}$ and $f \in L_{1}(\mu)$ such that all further subsequences $\left(h_{m}\right)_{m}$ of $\left(g_{k}\right)_{k}$ satisfy

$$
\frac{1}{N} \sum_{m=1}^{N} h_{m} \underset{N}{\rightarrow} f \quad \text { almost everywhere. }
$$

This result became the archetype for what Chatterji [C2] in the early 1970 s called "the subsequence principle in probability theory". This heuristic principle led Chatterji [C1], [C2], [C3] (see also Gaposhkin [Ga]) to find subsequence versions of the central limit theorem and 
the law of the iterated logarithm, analogous to Komlós's subsequence version of the strong law.

Chatterji [C1] and Gaposhkin [Ga] extended Komlós's theorem to all $L_{p}$ spaces, for $0<p<2$. Aldous [A] and Berkes and Péter [B-P], amongst others, continued the investigation of the subsequence principle using the notion of an exchangeable sequence of random variables.

A recent extension of Komlós's theorem, due to N. J. Kalton, may be found in Godefroy [Go]. Kalton strengthens the conclusion of Komlós's theorem so that the Cesàro means converge almost everywhere and in weak $L_{1}$.

For other recent developments concerning Komlós's theorem and further references, we refer the reader to Balder [B1], [B2], [B3] and Trautner [T].

In this paper we show that every convex set $C$ in $L_{1}(\mu)$ that satisfies the conclusion of Komlós's theorem, must be $L_{1}$-norm bounded. To prove this we proceed by contradiction. We create a sliding hump sequence of functions on the domain $\Omega$, each a member of $C$, for which certain convex combinations have Cesàro averages with an $L_{0}$ limit that lies outside of $L_{1}(\mu)$.

Finally, we characterize those convex subsets of $L_{1}$ that are almost everywhere Cesàro compact in the sense of the conclusion of Komlós's theorem, using a result of Bukhvalov and Lozanovski [B-L].

I thank Joe Diestel, Nigel Kalton, Amine Khamsi and Anton Schep for helpful comments and suggestions. Thanks also to Catherine for typing the manuscript.

The author is grateful for the support of a University of Pittsburgh Internal Research Grant during part of the preparation of this paper.

1. Preliminaries and Komlós sets. $\mathbf{N}$ denotes the set of all positive integers, while "the scalars" refers to the real or complex numbers. For a Banach space $X, \mathbf{B}_{X}$ denotes the closed unit ball of $X$.

Throughout this paper $\Omega$ will be a non-empty set, $\Sigma$ a $\sigma$-algebra of subsets of $\Omega$, and $\mu$ will be a complete, positive, $\sigma$-finite, countably additive measure on $\Sigma . L_{p}(\mu)$ is the $F$-space or Banach space of all (equivalence classes of ) measurable functions $f: \Omega \rightarrow$ the scalars for which $\|f\|_{p}<\infty$,

$$
\begin{aligned}
\|f\|_{1} & :=\int_{\Omega}|f| d \mu, \\
\|f\|_{\infty} & :=\operatorname{ess-sup}\{|f(\omega)|: \omega \in \Omega\},
\end{aligned}
$$


and

$$
\|f\|_{0}:=\sum_{n=1}^{\infty} \frac{1}{2^{n}} \frac{1}{\mu\left(E_{n}\right)} \int_{E_{n}} \frac{|f|}{1+|f|} d \mu .
$$

Here $\left(E_{n}\right)_{n=1}^{\infty}$ is a $\Sigma$-partition of $\Omega$ into sets with $0<\mu\left(E_{n}\right)<\infty$, for each $n$. Such a $\Sigma$-partition exists as $\mu$ is $\sigma$-finite. If $\mu$ is finite we have the simpler definition,

$$
\|f\|_{0}:=\int_{\Omega} \frac{|f|}{1+|f|} d \mu .
$$

The $L_{0}(\mu)$-topology restricted to $L_{1}(\mu)$ will be called the topology of convergence locally in measure (clm); or the topology of convergence in measure $(\mathrm{cm})$ when $\mu$ is finite. $\theta$ will denote the zero element in $L_{1}(\mu)$.

1.1. Definition. A subset $S$ of $L_{0}(\mu)$ will be called a Komlós set if for every sequence $\left(f_{n}\right)_{n=1}^{\infty}$ in $S$, there exists a subsequence $\left(g_{k}\right)_{k=1}^{\infty}$ of $\left(f_{n}\right)_{n=1}^{\infty}$ and $f \in S$ such that for every subsequence $\left(h_{m}\right)_{m=1}^{\infty}$ of $\left(g_{k}\right)_{k=1}^{\infty}$

$$
\frac{1}{N} \sum_{m=1}^{N} h_{m} \underset{N}{\rightarrow} f \quad \text { almost everywhere. }
$$

Komlós showed that $\mathbf{B}_{L_{1}(\mu)}$ is a Komlós set.

Note that if $\left(f_{n}\right)_{n=1}^{\infty}$ is a sequence in $L_{0}(\mu)$ and $f_{n} \underset{n}{\rightarrow} f$ almost everywhere, then

$$
\frac{1}{N} \sum_{n=1}^{N} f_{n} \underset{N}{\rightarrow} f \quad \text { almost everywhere. }
$$

It follows that every clm-compact subset $S$ of $L_{0}(\mu)$ must be a Komlós set. Consequently, even when Komlós sets are contained in $L_{1}(\mu)$, they need not be $L_{1}$-norm bounded (see $\S 2$ for an example). Further, it is easy to check that Komlós sets are forced to be $L_{0}$-closed. So, the concept of a Komlós subset of $L_{1}$ lies strictly between that of a clm-closed set and a clm-compact set in $L_{1}$.

\section{Convex Komlós sets in $L_{1}$ are norm bounded.}

2.1. Theorem. Let $(\Omega, \Sigma, \mu)$ be a finite measure space. Suppose $C$ is a subset of $L_{1}(\mu)$ that is convex and a Komlós set. Then $C$ must be $\|\cdot\|_{1}$-bounded. 
Proof. Suppose, to get a contradiction, that $C$ fails to be norm bounded. Then there exists a sequence $\left(g_{n}\right)_{n=1}^{\infty}$ in $C$ such that $\left\|g_{n}\right\|_{1} \underset{n}{\rightarrow} \infty$.

By assumption, $C$ is a Komlós set. So, by passing to a subsequence if necessary, we may assume that there exists $g \in C$ such that

$$
\frac{1}{N} \sum_{m=1}^{N} h_{m} \underset{N}{\rightarrow} g \text { almost everywhere }
$$

for every subsequence $\left(h_{m}\right)_{m}$ of $\left(g_{n}\right)_{n}$.

Note that $C-g$ is another convex Komlós set in $L_{1}(\mu), \theta \in C-g$ and

$$
\frac{1}{N} \sum_{m=1}^{N}\left(h_{m}-g\right) \underset{N}{\rightarrow} \theta \quad \text { almost everywhere }
$$

for all subsequences $\left(h_{m}\right)_{m}$ of $\left(g_{n}\right)_{n}$. Clearly, by relabelling each $g_{n}-g$ as $g_{n}$ and $C-g$ as $C$, we have that the following is true. $C$ is a convex Komlós set in $L_{1}(\mu),\left(g_{n}\right)_{n}$ is a sequence in $C$ with $\left\|g_{n}\right\|_{1} \underset{n}{\rightarrow} \infty, \theta \in C$ and for every subsequence $\left(h_{m}\right)_{m}$ of $\left(g_{n}\right)_{n}$,

$$
\frac{1}{N} \sum_{m=1}^{N} h_{m} \underset{N}{\rightarrow} \theta \quad \text { almost everywhere. }
$$

We shall now use $\left(g_{n}\right)_{n}$ to construct another sequence $\left(f_{n}\right)_{n}$ in $C$ such that $f_{n} \underset{n}{\rightarrow} \theta$ almost everywhere and $\left\|f_{n}\right\|_{1} \underset{n}{\rightarrow} \infty$. Let $u_{1}:=1$ and $f_{1}:=g_{u_{1}}$. Since $\left\|g_{n}\right\|_{1} \underset{n}{\rightarrow} \infty$, there exists $u_{2} \in \mathbf{N}$ with $u_{2}>u_{1}$ such that

$$
\left\|g_{u_{2}}\right\|_{1}>\left\|g_{u_{1}}\right\|_{1}+2\left(2^{2}\right)
$$

Define $f_{2}$ by

$$
f_{2}:=\frac{1}{2}\left(g_{u_{1}}+g_{u_{2}}\right),
$$

$f_{2} \in C$ because $C$ is convex. Also,

$$
\left\|f_{2}\right\|_{1} \geq \frac{1}{2}\left(\left\|g_{u_{2}}\right\|_{1}-\left\|g_{u_{1}}\right\|_{1}\right)>\frac{1}{2} \cdot 2\left(2^{2}\right)=2^{2} .
$$

Next choose $u_{3} \in \mathbf{N}$ with $u_{3}>u_{2}$ and

$$
\left\|g_{u_{3}}\right\|_{1}>\left\|g_{u_{1}}\right\|_{1}+\left\|g_{u_{2}}\right\|_{1}+3\left(2^{3}\right)
$$


and define

$$
f_{3}:=\frac{1}{3}\left(g_{u_{1}}+g_{u_{2}}+g_{u_{3}}\right) .
$$

Then $f_{3} \in C$ and $\left\|f_{3}\right\|_{1}>2^{3}$.

Continuing inductively, we produce a subsequence $\left(g_{u_{n}}\right)_{n=1}^{\infty}$ of $\left(g_{n}\right)_{n}$ and a sequence $\left(f_{n}\right)_{n=1}^{\infty}$ in $C$ such that $\left\|f_{n}\right\|_{1} \rightarrow \infty$ and

$$
f_{n}=\frac{1}{n} \sum_{j=1}^{n} g_{u_{j}}, \quad \text { for all } n \in \mathbf{N} .
$$

From above, we know that $f_{n} \underset{n}{\rightarrow} \theta$ almost everywhere.

We will now inductively construct a strictly increasing sequence $\left(n_{k}\right)_{k=0}^{\infty}$ in $\mathbf{N}$, a nonincreasing sequence $\left(E_{n}\right)_{n=0}^{\infty}$ in $\Sigma$ and a sequence $\left(\delta_{k}\right)_{k=0}^{\infty}$ of positive real numbers with the following properties. $E_{1}=\Omega$; and for each $k \in \mathbf{N}$ statements (1) to (5) below are true.

(1) $\delta_{k}<\delta_{k-1} / 2$.

(2) For each $E \in \Sigma$ with $\mu(E)<\delta_{k}$, we have that $\int_{E}\left|f_{n_{k}}\right| d \mu<1$.

(3) $\left\|f_{n_{k}} \chi_{E_{k}}\right\|_{1}>2^{k}(2+\mu(\Omega))$.

(4) $\left\|f_{n} \chi_{E_{k-1} \backslash E_{k}}\right\|_{\infty}<1$, for all $n \geq n_{k}$.

(5) $\mu\left(E_{k}\right)<\delta_{k-1}$.

Define $E_{0}:=\Omega, \delta_{0}:=2 \mu(\Omega)$ and $n_{0}:=1$. Next define $E_{1}:=\Omega$. Since $\left\|f_{n}\right\|_{1} \underset{n}{\rightarrow} \infty$, we can choose $n_{1} \in \mathbf{N}$ so large that $n_{1}>n_{0}$,

$$
\begin{aligned}
& \left\|f_{n_{1}} \chi_{E_{1}}\right\|_{1}>2^{1}(2+\mu(\Omega)), \quad \text { and } \\
& \left\|f_{n} \chi_{E_{0} \backslash E_{1}}\right\|_{\infty}<1, \quad \text { for all } n \geq n_{1} .
\end{aligned}
$$

By the absolute continuity of the measure $\left|f_{n_{1}}\right| d \mu$ with respect to $\mu$, there exists $\delta_{1} \in(0, \mu(\Omega))$ such that for every $E \in \Sigma$ with $\mu(E)<\delta_{1}$, we have

$$
\int_{E}\left|f_{n_{1}}\right| d \mu<1
$$

Of course, $\mu\left(E_{1}\right)<\delta_{0}$.

Fix $m \in \mathbf{N}$ with $m>1$. Suppose that we have constructed a strictly increasing sequence $\left(n_{k}\right)_{k=0}^{m-1}$ in $\mathbf{N}$, a non-increasing sequence $\left(E_{k}\right)_{k=0}^{m-1}$ in $\Sigma$ and a sequence $\left(\delta_{k}\right)_{k=0}^{m-1}$ of positive real numbers, such that statements (1) to (5) are true for each $k \in\{1, \ldots, m-1\}$. We know that $f_{n} \rightarrow \theta$ almost everywhere on $E_{m-1}$. So we can find, with the aid of Egoroff's theorem, $E_{m} \in \Sigma$ with $E_{m} \subseteq E_{m-1}$, such that

$$
\mu\left(E_{m}\right)<\delta_{m-1} \text { and }\left\|f_{n} \chi_{E_{m-1} \backslash E_{m}}\right\|_{\infty} \underset{n}{\rightarrow} 0 .
$$


But statement (4) is true for each $k \in\{1, \ldots, m-1\}$; and hence we see that

$$
\left\|f_{n} \chi_{\Omega \backslash E_{m-1}}\right\|_{\infty}<1, \quad \text { for all } n \geq n_{m-1} \text {. }
$$

Since $\left\|f_{n}\right\|_{1} \underset{n}{\rightarrow} \infty$, it follows that

$$
\sup _{n \in \mathbf{N}}\left\|f_{n} \chi_{E_{m}}\right\|_{1}=\infty
$$

Choose $n_{m} \in \mathbf{N}$ with $n_{m}>n_{m-1}$, such that

$$
\begin{aligned}
& \left\|f_{n_{m}} \chi_{E_{m}}\right\|_{1}>2^{m}(2+\mu(\Omega)), \quad \text { and } \\
& \left\|f_{n} \chi_{E_{m-1} \backslash E_{m}}\right\|_{\infty}<1, \quad \text { for all } n \geq n_{m} .
\end{aligned}
$$

Now, the measure $\left|f_{n_{m}}\right| d \mu$ is absolutely continuous w.r.t. $\mu$. Therefore there exists $\delta_{m}>0$ satisfying $\delta_{m}<\delta_{m-1} / 2$; and such that for every $E \in \Sigma$ with $\mu(E)<\delta_{m}$, we have that

$$
\int_{E}\left|f_{n_{m}}\right| d \mu<1
$$

Our inductive construction is complete.

For convenience, let us relabel each $f_{n_{k}}$ as $f_{k}$. We note that statements (2), (3) and (4) above still hold true, with $n_{k}$ replaced everywhere by $k$. We will refer to (2), (3) and (4), modified in this way, as $(2)^{*},(3)^{*}$ and $(4)^{*}$ respectively.

For each $k \in \mathbf{N}$, define

$$
\psi_{k}:=\sum_{j=1}^{k} \frac{1}{2^{j}} f_{j}
$$

Since $\theta \in C$, each $\psi_{k} \in \operatorname{co}(C)=C$. Also define, for every $m \in \mathbf{N}$,

$$
\varphi_{m}:=\left(\frac{1}{2^{m}}\left|f_{m}\right|-\sum_{j=1}^{m-1} \frac{1}{2^{j}}\left|f_{j}\right|-1\right) \chi_{E_{m} \backslash E_{m+1}} .
$$

$\left(\psi_{k}\right)_{k=1}^{\infty}$ is a sequence in $C$, which is a Komlós set in $L_{1}(\mu)$. So there exists a subsequence $\left(\psi_{k_{l}}\right)_{l=1}^{\infty}$ of $\left(\psi_{k}\right)_{k=1}^{\infty}$ and $q \in C$ such that

$$
q_{N}:=\frac{1}{N} \sum_{l=1}^{N} \psi_{k_{l}} \rightarrow{ }_{N} q \text { almost everywhere. }
$$


Moreover, note that $q \in C \subseteq L_{1}(\mu)$; so that

$(\mathbf{\$})$

$$
\|q\|_{1}<\infty
$$

Let $k_{0}:=0$. It is simple to verify that for all $N \in \mathbf{N}$,

$$
q_{N}=\sum_{j=1}^{N} \frac{N-j+1}{N} \sum_{t=k_{j-1}+1}^{k_{j}} \frac{1}{2^{t}} f_{t} .
$$

In the calculations below, when we have a pointwise inequality between two measurable functions, we mean that the inequality holds almost everywhere.

Fix $m \in \mathbf{N}$ and consider $E_{m} \backslash E_{m+1}$. Note that there is a unique $i \in \mathbf{N}$ such that $k_{i-1}<m \leq k_{i}$. Next fix $N \in \mathbf{N}$ with $N \geq i$. By property $(4)^{*}$ above, $\left|f_{j}\right|<1$ on $E_{m} \backslash E_{m+1}$, for all $j \geq m+1$. Temporarily, let $c_{m}:=\chi_{E_{m} \backslash E_{m+1}}$. Then,

$$
\begin{aligned}
\left|q_{N} c_{m}\right| & =\mid\left(\sum_{1 \leq j \leq N, j \neq i} \frac{N-j+1}{N} \sum_{t=k_{j-1}+1}^{k_{j}} \frac{1}{2^{t}} f_{t}\right. \\
& \left.+\frac{N-i+1}{N} \sum_{t=k_{i-1}+1}^{k_{i}} \frac{1}{2^{t}} f_{t}\right) c_{m} \mid \\
& \geq\left(\frac{N-i+1}{N} \frac{1}{2^{m}}\left|f_{m}\right|-\sum_{1 \leq t<m} \frac{1}{2^{t}}\left|f_{t}\right|-\sum_{m<t \leq k_{N}} \frac{1}{2^{t}}\left|f_{t}\right|\right) c_{m} \\
& \geq \frac{-(i-1)}{N} \frac{1}{2^{m}}\left|f_{m}\right| c_{m}+\varphi_{m}+c_{m}-\left(\sum_{m<t \leq k_{N}} \frac{1}{2^{t}}\right) c_{m} \\
& \geq \varphi_{m}-\frac{i-1}{N} \frac{1}{2^{m}}\left|f_{m}\right| c_{m} .
\end{aligned}
$$

Thus, we have shown the following.

(\$) For all $m \in \mathbf{N}$, there exists $i \in \mathbf{N}$ such that for all $N \in \mathbf{N}$ with $N \geq i$,

$$
\left|q_{N} \chi_{E_{m} \backslash E_{m+1}}\right| \geq \varphi_{m}-\frac{i-1}{N} \frac{1}{2^{m}}\left|f_{m}\right| \chi_{E_{m} \backslash E_{m+1}} .
$$


Again fix $m \in \mathbf{N}$. We see that

$$
\begin{aligned}
\int_{\Omega} \varphi_{m} d \mu= & \frac{1}{2^{m}} \int_{E_{m} \backslash E_{m+1}}\left|f_{m}\right| d \mu \\
& -\sum_{j=1}^{m-1} \frac{1}{2^{j}} \int_{E_{m} \backslash E_{m+1}}\left|f_{j}\right| d \mu-\mu\left(E_{m} \backslash E_{m+1}\right) \\
= & \frac{1}{2^{m}}\left\|f_{m} \chi_{E_{m}}\right\|_{1}-\frac{1}{2^{m}} \int_{E_{m+1}}\left|f_{m}\right| d \mu \\
& -\sum_{j=1}^{m-1} \frac{1}{2^{j}} \int_{E_{m} \backslash E_{m+1}}\left|f_{j}\right| d \mu-\mu\left(E_{m} \backslash E_{m+1}\right) .
\end{aligned}
$$

$\mu\left(E_{m+1}\right)<\delta_{m}$, from (5); and so by $(2)^{*}$,

$$
\int_{E_{m+1}}\left|f_{m}\right| d \mu<1 .
$$

Also, by (5) and (1) we have that for all $j \in\{1, \ldots, m-1\}$,

$$
\mu\left(E_{m} \backslash E_{m+1}\right) \leq \mu\left(E_{m}\right)<\delta_{m-1} \leq \delta_{j} ;
$$

and consequently from $(2)^{*}$,

$$
\int_{E_{m} \backslash E_{m+1}}\left|f_{j}\right| d \mu<1 .
$$

Using (3)* above,

$$
\begin{aligned}
\int_{\Omega} \varphi_{m} d \mu & >\frac{1}{2^{m}}\left\|f_{m} \chi_{E_{m}}\right\|_{1}-\frac{1}{2^{m}}-\sum_{j=1}^{m-1} \frac{1}{2^{j}}-\mu(\Omega) \\
& >\frac{1}{2^{m}} 2^{m}(2+\mu(\Omega))-1-\mu(\Omega)=1 .
\end{aligned}
$$

In summary,

$$
\int_{\Omega} \varphi_{m} d \mu>1, \quad \text { for all } m \in \mathbf{N}
$$

We now estimate $\|q\|_{1}$ from below. Fix $m \in \mathbf{N}$. By (\$), there exists $i \in \mathbf{N}$ such that for all $N \in \mathbf{N}$ with $N \geq i$, $\left|q_{N}(\omega)\right| \geq \varphi_{m}(\omega)-\frac{i-1}{N} \frac{1}{2^{m}}\left|f_{m}(\omega)\right|, \quad$ for almost all $\omega \in E_{m} \backslash E_{m+1}$.

From $(\diamond)$, we therefore have that

$$
|q(\omega)| \geq \varphi_{m}(\omega), \quad \text { for almost all } \omega \in E_{m} \backslash E_{m+1} .
$$


$E_{1}=\Omega$, and $\mu\left(E_{m}\right) \underset{m}{\rightarrow} 0$, by (1) and (5). Thus, $\left(E_{m} \backslash E_{m+1}\right)_{m=1}^{\infty}$ is a $\Sigma$-partition of $\Omega$. Consequently, using $(\$)$ and $(\nabla)$, we are led to the following contradiction.

$$
\begin{aligned}
\infty>\|q\|_{1} & =\sum_{m=1}^{\infty} \int_{E_{m} \backslash E_{m+1}}|q(\omega)| d \mu(\omega) \geq \sum_{m=1}^{\infty} \int_{E_{m} \backslash E_{m+1}} \varphi_{m}(\omega) d \mu(\omega) \\
& =\sum_{m=1}^{\infty} \int_{\Omega} \varphi_{m} d \mu \geq \sum_{m=1}^{\infty}(1)^{m}=\infty
\end{aligned}
$$

The previous theorem extends to the case where $\mu$ is a $\sigma$-finite measure. The proof below is simpler than our original one. It was suggested by Anton Schep.

2.2. Theorem. Let $(\Omega, \Sigma, \mu)$ be a $\sigma$-finite measure space. Let $C$ be a convex Komlós set in $L_{1}(\mu)$. Then $C$ must be norm bounded.

Proof. Fix $g \in L_{1}(\mu)$ such that

$$
g(\omega)>0, \quad \text { for all } \omega \in \Omega .
$$

Such a $g$ exists because $\mu$ is $\sigma$-finite. Define the finite measure $\nu$ by $d \nu:=g d \mu$, and define the linear isometry $T$ from $L_{1}(\mu)$ onto $L_{1}(\nu)$ by

$$
T f:=f g^{-1}, \quad \text { for all } f \in L_{1}(\mu) .
$$

Since $\mu$ and $\nu$ have the same sets of measure zero, it is easy to see that a subset $C$ of $L_{1}(\mu)$ is a Komlós set if and only if $T(C)$ is a Komlós set in $L_{1}(\nu)$. By Theorem 2.1,T(C) is $L_{1}(\nu)$-norm bounded; and consequently $C$ is $L_{1}(\mu)$-norm bounded.

Note that every clm-compact subset of $L_{1}$ is automatically a Komlós set. So the example

$$
C:=\left\{n^{2} \chi_{[0,1 / n]}: n \in \mathbf{N}\right\} \cup\{0\}
$$

is a Komlós set in $L_{1}[0,1]$ that fails to be $L_{1}$-norm bounded.

We also remark that a corollary to Theorem 2.1 is that every clmcompact, convex subset of $L_{1}(\mu)$ must be $L_{1}$-norm bounded. This is a result of Khamsi and Turpin [K-T], that can be generalized to the setting of a large class of tvs topologies $\tau$ on a Banach space $X$ (see, for example, Khamsi [Kh]).

3. A second dual characterization of Komlós convex sets in $L_{1}$. In this section the symbol $\cong$ will denote isometric isomorphism between 
Banach spaces. Let $j$ be the natural embedding of $L_{1}$ into $L_{1}^{* *}$. It is a fact that

$$
L_{1}^{* *}=j\left(L_{1}\right) \oplus_{1} S
$$

for some subspace $S$ of $L_{1}^{* *}$. Indeed, $L_{1}^{*} \cong L_{\infty}(\mu)$ and so $L_{1}^{* *} \cong$ $L_{\infty}^{*}$, which is isometrically isomorphic to the space of all bounded, finitely additive measures on $\Sigma$ that vanish on $\mu$-null sets. Hence, by the Yoshida-Hewitt decomposition theorem [Y-H] and the RadonNikodým theorem,

$$
L_{\infty}^{*} \cong L_{1} \oplus_{1} p f a(\mu),
$$

where $p f a(\mu)$ denotes the space of all bounded, purely finitely additive measures on $\Sigma$ that vanish on $\mu$-null sets. We identify $p f a(\mu)$ with a subspace $S$ of $L_{1}^{* *}$, and we denote by $P$ the natural projection of $L_{1}^{* *}$ onto $j\left(L_{1}\right)$.

Recall the following result, which we will use to establish Theorem 3.1 below.

Theorem (Bukhvalov and Lozanovski [B-L] Theorem 1). Let $C$ be a convex subset of $L_{1}(\mu)$ and let $W$ be the weak ${ }^{*}$-closure of $j(C)$ in $L_{1}^{* *}$.

(a) If $C$ is clm-closed then $P(W)=j(C)$.

(b) If $C$ is $L_{1}$-norm bounded and $P(W)=j(C)$ then $C$ is clmclosed.

3.1. THEOREM. Let $C$ be a convex subset of $L_{1}(\mu)$ and $W$ be the weak*-closure of $j(C)$ in $L_{1}^{* *}$. Then the following statements are equivalent.

(a) $C$ is a Komlós set.

(b) $C$ is $L_{1}$-norm bounded and clm-closed.

(c) $C$ is $L_{1}$-norm bounded and $P(W)=j(C)$.

Proof. (a) $\Rightarrow(\mathrm{b})$. By Theorem 2.2, $C$ is $L_{1}$-norm bounded. Moreover, Komlós sets are clm-closed, as we observed above.

(b) $\Rightarrow($ a $)$. Fix $\left(f_{n}\right)_{n=1}^{\infty}$ in $C$. By Komlós's theorem [Ko], there exists a subsequence $\left(g_{k}\right)_{k=1}^{\infty}$ of $\left(f_{n}\right)_{n=1}^{\infty}$ and $f \in L_{1}(\mu)$, such that for all subsequences $\left(h_{m}\right)_{m=1}^{\infty}$ of $\left(g_{k}\right)_{k=1}^{\infty}$ we have

$$
q_{N}:=\frac{1}{N} \sum_{m=1}^{N} h_{m} \underset{N}{\rightarrow} f \text { almost everywhere. }
$$

$C$ is convex, and hence each $q_{N} \in C$. But $C$ is clm-closed and consequently, $f \in C$.

(b) $\Leftrightarrow($ c). This follows from [B-L] Theorem 1 . 


\section{REFERENCES}

[A] D. J. Aldous, Limit theorems for subsequences of arbitrarily-dependent sequences of random variables, Z. Wahrsch. verw. Gebiete, 40 (1977), 59-82.

[B1] E. J. Balder, Infinite-dimensional extension of a theorem of Komlós, Probab. Theory Related Fields, 81 (1989), 185-188.

[B2] _ On uniformly bounded sequences in Orlicz spaces, Bull. Australian Math. Soc., 41 (1990), 495-502.

[B3] _ New sequential compactness results for spaces of scalarly integrable functions, J. Math. Anal. Appl., 151 (1990), 1-16.

[B-P] I. Berkes and E. Péter, Exchangeable random variables and the subsequence principle, Probab. Theory Related Fields, 73 (1986), 395-413.

[B-L] A. V. Bukhvalov and G. Lozanovski, On sets closed in measure in spaces of measurable functions, Trans. Moscow Math. Soc., 2 (1978), 127-148. (In Russian: Trudy Moskov. Mat. Obshch 34 (1977).)

[C1] S. D. Chatterji, A general strong law, Invent. Math., 9 (1970), 235-245.

[C2] $\quad$ A principle of subsequences in probability theory: the central limit theorem, Adv. in Math., 13 (1974), 31-54.

[C3] _ A subsequence principle in probability theory II. The law of the iterated logarithm, Invent. Math., 25 (1974), 241-251.

[Ga] V. F. Gaposhkin, Convergence and limit theorems for sequences of random variables, Theory Probab. Appl., 17 (1972), 379-400.

[Go] G. Godefroy, On Riesz subsets of abelian discrete groups, Israel J. Math., 61 (1988), 301-331.

[Kh] M. A. Khamsi, Note on a fixed point theorem in Banach lattices, preprint, (1990).

[K-T] M. A. Khamsi and Ph. Turpin, Fixed points of nonexpansive mappings in Banach lattices, Proc. Amer. Math. Soc., 105 (1989), 102 110.

[Ko] J. Komlós, A generalization of a problem of Steinhaus, Acta Math. Hungar., 18 (1967), 217-229.

[T] R. Trautner, A new proof of the Komlós-Révész-theorem, Probab. Theory Related Fields, 84 (1990), 281-287.

[Y-H] K. Yoshida and E. Hewitt, Finitely additive measures, Trans. Amer. Math. Soc., 72 (1952), 46-66.

Received October 3, 1991.

UNIVERSITY OF PitTsBurgh

Pittsburgh, PA 15260 



\section{PACIFIC JOURNAL OF MATHEMATICS}

Founded by

E. F. BECKENBACH (1906-1982) F. Wolf (1904-1989)

\section{EDITORS}

\author{
V. S. VARADARAJAN \\ (Managing Editor) \\ University of California \\ Los Angeles, CA 90024-1555 \\ vsv@math.ucla.edu \\ F. Michael Christ \\ University of California \\ Los Angeles, CA 90024-1555 \\ christ@math.ucla.edu
}

\section{Herbert Clemens}

University of Utah

Salt Lake City, UT 84112

clemens@math.utah.edu

\author{
ThOMAs ENRIGHT \\ University of California, San Diego \\ La Jolla, CA 92093 \\ tenright@ucsd.edu \\ Nicholas Ercolani \\ University of Arizona \\ Tucson, AZ 85721 \\ ercolani@math.arizona.edu \\ R. FINN \\ Stanford University \\ Stanford, CA 94305 \\ finn@gauss.stanford.edu \\ VAUGHAN F. R. Jones \\ University of California \\ Berkeley, CA 94720 \\ vfr@math.berkeley.edu
}

STEVEN KeRCKHOFF

Stanford University

Stanford, CA 94305

spk@gauss.stanford.edu

Martin ScharlemanN

University of California

Santa Barbara, CA 93106

mgscharl@henri.ucsb.edu

Harold Stark

University of California, San Diego

La Jolla, CA 92093

\section{SUPPORTING INSTITUTIONS}

UNIVERSITY OF ARIZONA

UNIVERSITY OF BRITISH COLUMBIA

CALIFORNIA INSTITUTE OF TECHNOLOGY

UNIVERSITY OF CALIFORNIA

UNIVERSITY OF MONTANA

UNIVERSITY OF NEVADA, RENO

NEW MEXICO STATE UNIVERSITY

OREGON STATE UNIVERSITY
UNIVERSITY OF OREGON

UNIVERSITY OF SOUTHERN CALIFORNIA

STANFORD UNIVERSITY

UNIVERSITY OF HAWAII

UNIVERSITY OF UTAH

WASHINGTON STATE UNIVERSITY

UNIVERSITY OF WASHINGTON 


\section{PACIFIC JOURNAL OF MATHEMATICS}

Volume 159 No. $1 \quad$ May 1993

An application of the very weak Bernoulli condition for amenable groups

SCOT ROBERT ADAMS and JEFFREY EDWARD STEIF

An application of homogenization theory to harmonic analysis on solvable Lie groups of polynomial growth

G. Alexopoulos

The standard double soap bubble in $\mathbf{R}^{2}$ uniquely minimizes perimeter

Joel Foisy, Manuel Alfaro Garcia, JefFrey FARlowe

Brock, NiCKELOUS Hodges and JASON ZimbA

Pseudo regular elements and the auxiliary multiplication they induce

BARRY E. JOHNSON

A converse to a theorem of Komlós for convex subsets of $L_{1}$

CHRISTOPHER JOHN LENNARD

General Kac-Moody algebras and the Kazhdan-Lusztig conjecture

WAYNE L. NEIDHARDT

The flow space of a directed $G$-graph

WILLIAM LINDALL PASCHKE

Primitive ideals and derivations on noncommutative Banach algebras

MARK PHILLIP THOMAS

Equivariant Nielsen numbers

PETER N-S WONG

Volumes of tubular neighbourhoods of real algebraic varieties

RichaRd ALEXANDER WONGKEW

The intrinsic group of Majid's bicrossproduct Kac algebra 